\title{
Kommentierte Buchanzeigen / Book Review
}

Luuk van Middelaar: The Passage to Europe. How a Continent Became a Union. New Haven/London: Yale University Press 2013.

Es bedurfte erstaunlich vieler Jahre, bis dieses Buch, 2009 in einer holländischen Ausgabe vorgelegt, seine Übersetzung und damit einen breiteren Leserkreis fand - eine angesichts der Ereignisse, die die europäische Entwicklung zwischenzeitlich prägten, bedauerliche Verzögerung. Dies umso mehr, als der Autor sich seiner Analyse des europäischen Entwicklungswegs zwar als Historiker nähert, erkennbar aber auch von beträchtlichen und zeitnahen Erfahrungen in wie mit Brüsseler Einrichtungen profitiert. Das Selbstbild ist das eines empirisch informierten politischen Philosophen, dessen Zugänge sich von gängigen Analyseund Interpretationsmustern unterscheiden: „European momentum originates in an unpredictable series of decisions, often by national leaders grappling with events both at home and abroad and forced to deal with them jointly, sometimes with obvious reluctance. This political interplay offers a more plausible explanation than either the pseudo-logic of integration theory and federalist teleology, or the Eurosceptic world-view of evil conspiracies and Brussels-led schemes to induce foreign rule." Dies führt vM. zu einer Reihe untypischer (und erfrischender) Sichtweisen, so etwa beim Ersatz der ubiquitären Metapher einer ever closer Union durch ein zurückgenommenes advancing together, wobei er „klassische“ Kategorien wie foundation, change, representation, legitimacy, responsibility, events and freedom an die Stelle der so verbrauchten wie analytisch unzureichenden hard and soft power, eines multi-level governance oder eines behaupteten bzw. tatsächlichen democratic deficits setzt. In den drei Kapitelüberschriften kommt das bereits zum Ausdruck: The secret of the table:transition to majority, Vicissitudes of fortune: in the river of time und The quest for a public: winning applause. Zudem zahlt sich aus, dass Gremienerfahrungen auf nationalstaatlicher (niederländischer) und europäischer Ebene einbezogen werden; seit geraumer Zeit fungiert der Autor zudem als speech writer für den Ratspräsidenten Van Rompuy. Das Buch ist all Denjenigen zu empfehlen, die einer breiten, aber analytisch anspruchsvollen Versicherung der europäischen Idee bedürfen, zumal das europapolitische Wollen hier eine erfahrungsgesättigte Ergänzung um das unabdingbare Können (von Einrichtungen und Akteuren) erfährt. 
Azar Gat (mit Alexander Yakobson): Nations. The Long History and Deep Roots of Political Ethnicity and Nationalism. Cambridge: Cambridge University Press 2013.

G., Professur für Political Science an der Universität Tel Aviv, präsentiert in seiner anthropologisch ausgerichteten Weltgeschichte Europa vor allem im Vergleich zu Asien als einen nationalpolitischen Spätentwickler. Die geläufige Überzeugung, europäische Gesellschaften hätten als erste Nationen und Nationalstaaten ausgeformt, sei ,fundamentally untrue“. Dieses Missverständnis beruhe darauf, dass Europa die älteren Entwicklungen in anderen Regionen der Welt gänzlich überschattet habe, als es im 19. Jh. zum global überlegenen Modernisierungsraum aufstieg. - G. geht es darum, politisch wirksame Konstanten in der menschlichen Geschichte zu erkennen. Seine Studie führt deshalb von den Anfängen menschlichen Lebens bis zur Europäischen Union, die er auf der Suche nach ,gene-culture interactions“ in eine provozierende Perspektive rückt. In seinem anthropologischen Blick wird die EU ebenso wie Stammesgesellschaften oder vormoderne Staaten auf einige Kernlinien reduziert, um die Verbindungen zwischen ,kinship, culture, and identity formation“ zu erkennen. G. legt die Zukunft nicht an die Kette der Vergangenheit, doch Ethnizität und nationale Bindungen gelten ihm als Prägekräfte, die seit jeher alle Formen von Staat und Gesellschaft gestaltet haben. Wie stark sie auch heute noch unsere Vorstellungen bestimmen, dokumentiert $\mathrm{Y}$. in einem eigenen Kapitel an Verfassungen und anderen normativen Texten der Gegenwart. - Die EU sei historisch ohne Vorläufer, und doch konstatiert G. in ihr einen ,ethnic revival“; nicht Nationalstaaten erodieren, sondern ihre Souveränität. Ob daraus eine supranationale Identität hervorgehen könne, vermag er mit seinem anthropologischen Geschichtsmodell nicht zu prognostizieren. Doch es legt nahe, mit sehr langen Zeitspannen zu rechnen, wenn überzeitlich wirksame soziale Bindekräfte auf eine neue institutionelle Ordnung ausgerichtet werden sollen. G.s Geschichtsanalyse bietet dazu keine konkreten Handlungshilfen, regt aber an, über die Größe der Aufgabe nachzudenken.

DL 
Sebastian Conrad: Globalgeschichte. Eine Einführung. München: C.H. Beck 2013. / Emily S. Rosenberg (Hg.): Weltmärkte und Weltkriege 1870 - 1945. Bd. 1 der ,Geschichte der Welt“, hgg. v. Akira Iriye/Jürgen Osterhammel. München: C.H. Beck 2012 (zugleich, in englischer Sprache: Cambridge [M.A.]: Harvard University Press/The Belknap Press 2012).

Globalgeschichten gilt seit geraumer Zeit nicht nur das Interesse der Historiker, sondern auch all jener Disziplinen, für die Raum-/Zeitbezüge von Bedeutung sind. Kaum ist die Diskussion im deutschsprachigen Raum nicht zuletzt durch Osterhammel et al. eröffnet (s. ZSE 8/1 (2001), S. 153), kommt es zu diversen epochenspezifischen wie sektoralen Ausdifferenzierungen und findet sich sogar eine (zweifelsfrei sehr frühe) Einführung in die Idee der Globalgeschichte. Letztere, mithin eine „Geschichte der Weltgeschichte“, legt C. vor, im Rahmen einer knappen gut lesbaren Zusammenfassung, die den Entwicklungsprozess nachzeichnet, theoretische Zugänge, Untersuchungsansätze und verfolgenswerte Kategorien benennt sowie schließlich Kontroversen ausweist. Der Autor ist dabei klug beraten, das abschließende Kapitel als „Globalgeschichte in Aktion“ zu kennzeichnen, mithin auch und gerade in diesem Kontext einem längeren Atem, einer longue durée, das Wort zu reden. Nur so auch dürfte den bereits erkennbaren Themenzyklen zu begegnen sein, trennt man zudem die Spreu vom Weizen. In einer ersten vorsichtigen definitorischen Annäherung bezeichnet C. die Globalgeschichte als eine Form der historischen Analyse, bei der Phänomene, Ereignisse oder auch Prozesse in globale Kontexte eingeordnet werden. Damit sei nicht notwendigerweise gemeint, dass die Untersuchung auf den ,ganzen Erdball“ ausgedehnt wird; bei vielen Themen werden die Bezugspunkte vielmehr begrenzter sein. Das heißt dann aber auch, dass die meisten globalgeschichtlichen Ansätze nicht versuchen, das etablierte nationalgeschichtliche Paradigma durch eine abstrakte ,Totalität der Welt“" zu ersetzen, gleichsam eine Totalgeschichte des Globus schreiben zu wollen. Häufig ginge es eher um die Geschichtsschreibung begrenzter, also nicht-globaler Räume, freilich mit einem Bewusstsein für globale Zusammenhänge. Die Reichweite entsprechender Studien könne daher nach Thema und Fragestellung variieren, muss zudem keineswegs die ganze Welt umfassen; sie sei im Ergebnis auch nicht zwingend makrogeschichtlich ausgerichtet. Nimmt man solche Aussagen zum Maßstab für die vorliegende Literatur (ergänzt um die Einschätzung des Autors, nach der sich die „spannendsten Fragen“ häufig am Schnittpunkt globaler Prozesse und ihrer lokalen Manifestationen stellen), bleibt freilich das Problem der Abgrenzung. Ist Weltgeschichte nach diesem Verständnis dann nicht doch eher (simple) Maß- 
stabsvergrößerung, gleichsam eine Externalisierung lokaler, regionaler und nationalhistorischer Untersuchungen? Diese Einführung versucht, solche Fragen offenzuhalten, und das aus gutem Grund. - Blickt man etwa auf das große gemeinsam von C.H. Beck und der Harvard University Press verantwortete Vorhaben „Geschichte der Welt/History of the World“, so verweist dessen erster Ertrag, die Analyse der Weltmärkte und Weltkriege von 1870 bis 1945, auf die Handlungsmöglichkeiten wie die Grenzen eines entsprechenden Unterfangens. So ist hier der jeweilige Kontext zwar unbezweifelbar ,globalisiert“, dominieren letztlich aber doch sektorale Sichtweisen, die sich so auch anderenorts und unter anderer Flagge finden. Zudem wird erkennbar, wie schwer sich die unterschiedlichen Zugänge einer auch nur ansatzweisen Generalisierung öffnen. Letztlich setzt sich der Band aus einer Reihe von (vorzüglichen) Einzelpublikationen zusammen, die sich einer den Namen verdienenden vergleichenden Analyse aber versperren. Damit verbindet sich dann allerdings die Frage nach dem Erkenntnisfortschritt: Gewinnt die Globalgeschichte über Publikationen dieser Art tatsächlich an Terrain oder ist man eher auf einem gleichsam kompilatorischen Weg, der im Aufaddieren unterschiedlichster Zugänge in eine „Weltgeschichte“ münden soll?

M. Rainer Lepsius: Institutionalisierung politischen Handelns. Analysen zur DDR, Wiedervereinigung und Europäischen Union. Wiesbaden: Springer VS 2013.

Dieser Band vereint seit 1995 erschienene Aufsätze, mit denen L. die soziologische Institutionenanalyse theoretisch fundiert und ihre Anwendung in den Themenfeldern „Institutionenordnung in der DDR“, „Institutionelle Formungen der Wiedervereinigung“ und „Institutionenbildung in der Europäischen Union“" (so die Kapitelüberschriften) erprobt hat. Hinzu kommt als historischer Exkurs eine Studie, die im Halleschen Pietismus die ,Mentalität des preußisch-deutschen Wohlfahrtsstaates“ konstitutiv angelegt sieht. - In allen Bereichen geht es um Prozesse der Institutionalisierung von Ordnungsvorstellungen und deren Durchsetzung in ausdifferenzierten Handlungskontexten. Die vier Beiträge zur EU (erschienen zwischen 1999 und 2013) befragen die Entwicklung und die Entwicklungspotentiale der Union, indem sie deren Aufgabenerweiterung präzise den komplexen Prozessen der Institutionenbildung zuschreiben. Die EU als ,,politisch gewollter und rechtlich konstituierter, neuartiger und selbständiger Herr- 
schaftsverband“ befinde sich auf dem ,Weg zu einer Kompetenzkompetenz mit eigener politischer Identität“. Diese „Staatswerdung“ sollte aber nicht in Analogie zur Entstehung der europäischen Nationalstaaten gesehen werden. Es gehe nicht darum, ,nationale Identitäten“ zu homogenisieren, sondern die Rationalitätskriterien, die in den verschiedenen Tätigkeitsfeldern der EU ausgebildet werden, in die Lebenswelten und die ,kulturellen Selbstverständnisse“ der Mitgliedstaaten zu ,übersetzen“. Eine Verfassung als symbolisches Dach könne diese Aufgabe, Legitimität zu erzeugen und Identität zu stiften, nicht lösen. L. plädiert für eine Ausweitung der bisherigen ,paktierten Kooperation“ auf den „Sozialraum Europa“. Dazu müsse weder die Institutionenordnung der EU umgebaut, noch die der Mitgliedstaaten uniformiert werden. Erforderlich sei vielmehr eine gemeinsame ,Zielorientierung“, die verpflichtend vereinbart wird und für übereinstimmende Rationalitätskriterien in der Politik sorgt. - Mehr Europa, nicht mehr oder andere europäische Institutionen, auf dieses Plädoyer laufen L.s Analysen hinaus. Die „,verschränkte duale Legitimation“ der EU sei ,keine schwache Legitimität“, sondern sie fördere die Akzeptanz der „Völker Europas“.

DL

Benjamin Lahuse: Alles Recht geht vom Volksgeist aus. Friedrich Carl von Savigny und die moderne Rechtswissenschaft. Berlin: Nicolaische Verlagsbuchhandlung 2013.

Diese knappe Biographie Savignys aus der Feder eines jüngeren Rostocker Rechtswissenschaftlers verdient Aufmerksamkeit. Zum einen als (gut geschriebenes) Portrait des ,bedeutendsten deutschen Rechtsgelehrten“, der Generationen von Juristen in das Fach einführte und deren Denken formte, zum anderen als kritische Auseinandersetzung mit der Lehre vom Volksgeist als Grundlegung des modernen Rechts. Mit dieser Lehre entwarf Savigny bekanntlich ein Programm, mit dem er das alte Naturrecht zu überwinden und der Rechtswissenschaft den Weg in die Moderne zu bahnen suchte. Aber so revolutionär seine Wissenschaft auch war, politisch stand der vielfach privilegierte Adlige den Fortschritten seiner Zeit kritisch gegenüber. Das wirkt, der Analyse von L. zufolge, bis heute nach. Die Verwaltung der Gerechtigkeit ist noch immer eine elitäre Angelegenheit und ausgerechnet Savignys Lehre vom Volksgeist steht für eine Tradition, in der Gesetze nicht vom Volke kommen und sich nicht an das Volk richten. Den damit angesprochenen Zwiespalt dokumentiert der Autor 
nahezu vorbildlich, da er die biographischen Elemente der Darstellung mit einer knappen Geschichte der Rechtswissenschaft zu verbinden vermag. Doch was bleibt letztlich? „Bequemer Verehrungstrieb“ oder, zeithistorisch betrachtet, „der Durchbruch aus Norm und Disziplin“? Das Fazit L.s dürfte einige der Leser überraschen: „Nein, das Erbe einer historischen Größe wie Savigny kann man nicht im Konkreten suchen. Seine Prägungen der Gegenwart liegen im diffusen Bereich des Kulturellen, der zwar schwerer greifbar ist als die dogmatischen Spezifika seiner juristischen Alltagsarbeit, dafür aber in seinen Wirkungen deutlich tiefer geht. Die Nachwelt verdankt Savigny einen neuen Blick auf das Phänomen Recht, eine neue Sehweise, ein neues Paradigma. Unter seiner Führung wurde das Recht vom Himmel auf die Erde geholt, dem unergründlichen Willen der Natur entrissen und in Menschenhand gelegt - nicht in die Hände aller Menschen, aber in Juristenhände, immerhin. Ein bloß dienendes Handwerk stieg zu einem Gegenstand auf, der seinerseits eigenständige, wissenschaftliche, ästhetische, kulturelle Beschäftigung verdient. Nur deshalb konnte das 19. Jahrhundert zum Jahrhundert der Jurisprudenz werden, nur deshalb gab es einmal eine Zeit, in der Juristen Leistungen vollbracht haben, die man zum nationalen Kulturgut zählen kann.“

Maurizio Bach (Hg.): Der entmachtete Leviathan. Löst sich der souveräne Staat auf? Sonderband 5 der Zeitschrift für Politik. Baden-Baden: Nomos 2013.

Dieses Sonderheft der ZfP enttäuscht. Suggeriert der Titel der Publikation (ohne ein Fragezeichen) die „Entmachtung“ des Staates, eine These, der man sich aus gutem Grund zuwenden kann, stellt der Untertitel dies bereits wieder in Frage. Dieser Widerspruch zieht sich durch das gesamte Heft und wird in Ermanglung einer Zusammenfassung der höchst disparaten Beiträge auch nicht aufgelöst. So findet sich Allerlei zu behaupteten (aber eher selten belegten) Funktionsveränderungen der Staatlichkeit, gilt der Europäisierung und wohlfahrtsstaatlichen Entwicklungen gesondertes Interesse, findet sich aber nichts zu den sich damit verbindenden normativen und funktionalen Voraussetzungen wie Folgen. Dies ist umso bedauerlicher, als man damit weder dem Stand der empirisch-analytischen noch der vergleichenden Forschung gerecht wird, und auch nicht jener sich in den vergangenen Jahren intensivierenden Diskussion zum Verhältnis von „Nationalstaat und Europäischer Union“. Hierzu hätte es einer substantiellen Erörte- 
rung eben jener normativen wie funktionalen Vorrausetzungen künftigen öffentlichen Handelns bedurft, nicht nur gleichsam nach „oben“, also mit Blick auf Globalisierungs- und Europäisierungsprozesse, sondern auch nach „unten“, unter explizitem Einbezug der den politischen Willen konstituierenden Einrichtungen und der ihn umsetzenden Gebietskörperschaften. Angesichts des Standes der in diesem Kontext national wie international erkennbaren rechts-, wirtschafts- und sozialwissenschaftlichen Diskussion wird man weder vom ,entmachteten Leviathan“ noch von einer „Auflösung des souveränen Staates“ sprechen können.

\section{Am Rande oder: Zu guter Letzt / At long last}

Borgen - Gefährliche Seilschaften, 1. und 2. Staffel (deutschsprachig auf DVD erschienen bei WVG Medien GmbH, 2012/2013); 3. Staffel derzeit auf ARTE.

Die mediale Aufarbeitung grundlegender rechtlicher, wirtschaftlicher und vor allem politischer Prozesse stellt bekanntlich eine wichtige, ja unverzichtbare Aufgabe demokratischer Gemeinwesen dar. Blickt man dabei auf das hierzulande primäre Medium, das deutsche Fernsehen, sind die Vorbehalte seit langer Zeit Legion: zu punktuell, ,flach“ und nicht zuletzt angepasst wirkt das, was nicht nur die privaten, sondern auch die überaus großzügig alimentierten öffentlichrechtlichen Anbieter produzieren. So befinden sich die einstmals blühenden politischen Magazine in gleichsam ,freiem Fall“, reduziert sich die weitere Berichterstattung auf sehr basale Informationen im Rahmen von Nachrichtensendungen und kommt es lediglich aus Anlass von Wahlterminen zu etwas ausführlicheren, die Oberfläche freilich selten unterschreitenden Formaten. Politisches auch über andere Vermittlungsformen aufzubereiten, ist äußerst selten und von meist indiskutabler Qualität. - Anders das Ausland: Hier ist es derzeit das kleine Dänemark, das mit „Borgen“, dem Synonym für Christiansborg, dem Sitz der dänischen Regierung und des Parlaments, eine Produktion bereits in der dritten Staffel vorstellt, die man als exemplarische Aufklärung über politische Prozesse (im weitesten Sinne) verstehen kann. Es geht am Beispiel des Aufstiegs, der Regierungszeit und des Falls einer Ministerpräsidentin um ein ungeschminktes Bild auf den politischen Prozess, seine Einrichtungen und Akteure - weder übernoch unterzeichnet, in jeder Hinsicht lebensnah und vor allem in undogmatischer Absicht. Natürlich hat auch dieses Bravourstück ,politischer Berichterstattung“ ein Vorbild, das es aber weit übertrifft: „The West Wing“ (des Weißen Hauses) 
hieß jene Serie, die erstmals gekonnt über die Grundzüge des politischen Systems, hier der USA, informierte, allerdings im Rahmen der eher gewöhnungsbedürftigen Routinen des amerikanischen Fernsehens. „Borgen“ ist dem deutlich überlegen: aufgrund des wesentlich erweiterten Verständnisses des Politischen, seiner Verflechtung mit nahezu allen Arbeits- und Lebensbereichen und, nicht zuletzt, einer überragenden ästhetischen Qualität. Chapeau! 